\title{
Fritz John Duality in the Presence of Equality and Inequality Constraints
}

\author{
Iqbal Husain, Santosh K. Shrivastav \\ Department of Mathematics, Jaypee University of Engineering and Technology, Guna, India \\ Email: ihusain11@yahoo.com, santosh_00147@rediffmail.com
}

Received June 10, 2012; revised August 3, 2012; accepted August 10, 2012

\begin{abstract}
A dual for a nonlinear programming problem in the presence of equality and inequality constraints which represent many realistic situation, is formulated which uses Fritz John optimality conditions instead of the Karush-Kuhn-Tucker optimality conditions and does not require a constraint qualification. Various duality results, namely, weak, strong, strict-converse and converse duality theorems are established under suitable generalized convexity. A generalized Fritz John type dual to the problem is also formulated and usual duality results are proved. In essence, the duality results do not require any regularity condition if the formulations of dual problems uses Fritz John optimality conditions.
\end{abstract}

Keywords: Second-Order Invexity; Second-Order Pseudoinvexity; Second-Order Quasi-Invexity; Nonlinear Programming; Fritz John Type Dual

\section{Introduction}

Consider the following mathematical programming problems.

(NP): Minimize $f(x)$

Subject to

$$
g(x) \leq 0
$$

(NEP): Minimize $f(x)$

Subject to

$$
\begin{aligned}
& g(x) \leq 0 \\
& h(x)=0
\end{aligned}
$$

where $f: R^{n} \rightarrow R, g: R^{n} \rightarrow R^{m}$ and $h: R^{n} \rightarrow R^{k}$ are differentiable functions. The best-known necessary optimality conditions for the mathematical programming problem (NP) and (NEP) are Fritz John necessary optimality conditions and Karush-Kuhn-Tucker type optimality conditions. The Fritz John type [1] optimality condition which predates the Karush-Kuhn-Tucker type optimality conditions by a few years are more general in a sense. In order for Karush-Kuhn-Tucker type optimality conditions to hold, a constraint qualification or regularity condition on the constraint is required. On the other hand, no such constraint qualification is needed for Fritz John optimality conditions to hold.

Fritz John [2] established the following optimality conditions for (NP):

Proposition 1. (Fritz John type necessary conditions).
If $\bar{x}$ is an optimal solution of (NP), then there exist $\bar{r} \in R$ and a vector $\bar{y} \in R^{m}$ such that

$$
\begin{gathered}
\bar{r} \nabla f(\bar{x})+\bar{y}^{T} \nabla g(\bar{x})=0 \\
\bar{y}^{T} g(\bar{x})=0 \\
(\bar{r}, \bar{y}) \geq 0 \\
(\bar{r}, \bar{y}) \neq 0
\end{gathered}
$$

Using these optimality conditions, Weir and Mond [3] formulated the for Fritz John type dual $\left(F_{r} D\right)$ to (NP) and established usual duality theorems, this eliminating the requirement of a constraint qualification:

$\left(F_{r} D\right)$ : Maximize $f(u)$ Subject to

$$
\begin{gathered}
r \nabla f(u)+y^{T} \nabla g(u)=0 \\
y^{T} g(u) \geq 0 \\
(r, y) \geq 0 \\
(r, y) \neq 0 .
\end{gathered}
$$

Originally, Fritz John derived his optimality condition for the case of inequality constraint alone. If equality constraint are present in a mathematical programming problem and they are converted into two inequality constraints, then the Fritz John optimality conditions become useless because every feasible point satisfying them. Later Mangasarian and Fromovitz [4] derived necessary 
optimality condition for (NEP) without replacing an equality constraint by two inequalities and hence making it possible to handle equalities and inequalities together as many realistic problems contain both equality and inequality constraint. Mangasarian and Fromovitz [4] established the following Fritz John type optimality condition given in the following propositions:

Proposition 2. (Generalized Fritz John necessary optimality Conditions [4]):

If $\bar{x}$ is an optimal solution of (NEP), then there exist $\bar{r} \in R, \bar{y} \in R^{m}$ and $\bar{z} \in R^{k}$ such that

$$
\begin{gathered}
\bar{r} \nabla f(\bar{x})+\bar{y}^{T} \nabla g(\bar{x})+\bar{z}^{T} \nabla h(\bar{x})=0 \\
\bar{y}^{T} g(\bar{x})=0 \\
(\bar{r}, \bar{y}) \geq 0 \\
(\bar{r}, \bar{y}, \bar{z}) \neq 0
\end{gathered}
$$

\section{Sufficiency of Fritz John Optimality Conditions}

Before proceeding to the main results of our analysis we give the following definitions which are required for their validation.

1) The function $\phi: R^{n} \rightarrow R$ is strictly pseudoconvex on $R^{n}$ for all $x \neq u$,

$$
(x-u)^{T} \nabla \phi(u) \geq 0 \Rightarrow \phi(x)>\phi(u)
$$

Equivalently

$$
\phi(x) \leq \phi(u) \Rightarrow(x-u)^{T} \nabla \phi(u)<0
$$

2) For $y \in R^{m}$ and $\psi: R^{n} \rightarrow R^{m}, y^{T} \psi$ is said to be semi-strictly pseudoconvex if $y^{T} \psi$ is strictly pseudoconvex for all $y \geq 0, y \neq 0$.

Theorem 1. (Sufficient Optimality Conditions): Assume that

1) $f($.$) is pseudoconvex,$

2) $\bar{y}^{T} g($.$) is semi strictly pseudoconvex and$

3) $z^{T} h($.$) is quasiconvex,$

If there exist $\bar{x}, r \in R, y \in R^{m}$ and $z \in R^{k}$ such that (3)-(8) are satisfied, then $\bar{x}$ is an optimal solution of (NEP).

Proof: Suppose $\bar{x}$ is not optimal, i.e., and then there exists $x \neq \bar{x}$ Such that

$$
f(x)<f(\bar{x})
$$

Since $f($.$) is pseudoconvex, this implies$

$$
(x-\bar{x})^{T} \nabla f(\bar{x})<0
$$

and

$$
(x-\bar{x})^{T} \bar{r} \nabla f(\bar{x}) \leq 0
$$

with strict-inequality in the above if $\bar{r}>0$
Since $\bar{x}$ is feasible for $(N E P)$ we have

$$
y^{T} g(x) \leq \bar{y}^{T} g(\bar{x})
$$

Because of semi strict pseudoconvexity of $\bar{y}^{T} g($.$) ,$ This implies

$$
(x-\bar{x})^{T} \nabla \bar{y}^{T} g(\bar{x}) \leq 0
$$

With strict inequality with $y^{i}()>0,. i \in\{1,2,3, \cdots, m\}$.

Also $\bar{z} h(x)=\bar{z}^{T} h(\bar{x})$

Because of quasi-convexity of $z^{T} h($.$) at \bar{x}$,

$$
(x-\bar{x})^{T} \nabla z^{T} h(\bar{x}) \leq 0
$$

Combining (7), (8) and (9), we have

$$
(x-\bar{x})^{T}\left(\bar{r} \nabla f(\bar{x})+\nabla y^{T} g(\bar{x})+\nabla z^{T} h(\bar{x})\right)<0,
$$

Contradicting (3). Hence $\bar{x}$ is an optimal solution of (NEP).

\section{Fritz John Type Duality}

We propose the following dual $\left(F_{r} E D\right)$ to $(N E P)$, using Fritz John optimality conditions stated in the preceding section instead of Karush-Kuhn-Tucker conditions [5,6] and established duality results, thus the requirement of a constraint qualification [4] is eliminated:

Dual Problem:

$$
\begin{aligned}
& \left(F_{r} E D\right) \text { : Maximize } f(u) \\
& \text { Subject to } \\
& \nabla\left(r f(u)+y^{T} g(x)+z^{T} h(x)\right)=0 \\
& y^{T} g(u) \geq 0 \\
& z^{T} h(u) \geq 0 \\
& (r, y) \geq 0 \\
& (r, y, z) \neq 0
\end{aligned}
$$

Theorem 2. (Weak Duality): Assume that

$\left(A_{1}\right): x$ is feasible for (NEP) and $(u, r, y, z)$ is feasible for $\left(F_{r} E D\right)$.

$\left(A_{2}\right)$ : For all feasible $(x, u, r, y, z), f($.$) is pseu-$ doconvex, $y^{T} g($.$) is semi-strictly pseudoconvex and$ $z^{T} h($.$) is quasiconvex.$

Then

$$
\inf (N E P) \geq \sup \left(F_{r} E D\right)
$$

Proof: Suppose $f(x)<f(u)$ this, because of pseudoconvexity of $f($.$) yields (x-u)^{T} \nabla f(u)<0$, Multiplying this, by $r \geq 0$, We have

$$
(x-u)^{T} \nabla r f(u) \leq 0
$$

With strict inequality in (15) if $r>0$

From the Constraints of $(N E P)$ and $\left(F_{r} E D\right)$, we have 


$$
y^{T} g(x) \leq y^{T} g(u)
$$

which by semi-strictly pseudoconvexity of $y^{T} g($.$) im-$ plies

$$
(x-u)^{T} \nabla y^{T} g(u) \leq 0
$$

with strict inequality in (16) if $y_{i}>0, i \in(1,2,3, \cdots, m)$

As earlier $y^{T} h(x) \leq y^{T} h(u)$

This along with quasiconvexity of $y^{T} h($.$) implies$

$$
(x-u)^{T} \nabla y^{T} h(u) \leq 0
$$

Combining (15), (16), (17), we have

$$
(x-u)^{T} \nabla\left(r f(u)+y^{T} g(u)+z^{T} h(u)\right)<0
$$

Contradicting

$$
(x-u)^{T} \nabla\left(r f(u)+y^{T} g(u)+z^{T} h(u)\right)=0
$$

Hence $f(x) \geq f(u)$

This implies $\inf (N E P) \geq \sup \left(F_{r} E D\right)$.

Theorem 3. (Strong Duality):

If $\bar{x}$ is an optimal solution of $(N E D)$ then there exist $r \in R, y \in R^{m}$ and $z \in R^{k}$ such that $(\bar{x}, r, y, z)$ is feasible for $(N E D)$ and the corresponding values of $(N E P)$ and $\left(F_{r} E D\right)$ are equal. If, also $f$ is pseudoconvex, $y^{T} g($.$) is semi-strictly pseudoconvex and z^{T} h($.$) is$ quasi-convex, then $(\bar{x}, r, y, z)$ is an optimal solution of (NED).

Proof: Since $\bar{x}$ is an optimal solution of $(N E P)$, by Proposition 2. There exist $r \in R, y \in R^{m}$ and $z \in R^{k}$ such that

$$
\begin{gathered}
\nabla\left(r f(\bar{x})+y^{T} g(\bar{x})+z^{T} h(\bar{x})\right)=0, \\
y^{T} g(\bar{x})=0, \\
g(\bar{x}) \leq 0 \\
h(\bar{x})=0 \\
(r, y) \geq 0,(r, y, z) \neq 0
\end{gathered}
$$

This implies $(\bar{x}, r, y, z)$ is feasible for $\left(F_{r} E D\right)$. Equality of objective function of $(N E P)$ and $\left(F_{r} E D\right)$ is abovious optimality follows, in view of the hypothesis of the theorem1.

Theorem 4. (Strict Converse Duality): Assume that

1) $f($.$) is strictly pseudoconvex, y^{T} g($.$) is semi-$ strictly pseudoconvex and as $z^{T} h($.$) is quasiconvex and$

2) The problem $(N E P)$ has an optimal solution $\bar{x}$.

If $(\bar{u}, r, y, z)$ is an optimal solution of $\left(F_{r} E D\right)$, Then $\bar{u}=\bar{x}$ i.e. $\bar{u}$ is an optimal solution of (NEP).

Proof: We assume that $\bar{x} \neq \bar{u}$ and exhibit a contradiction, it follows from Proposition 2 that there exist $r \in R, y \in R^{m}$ and $z \in R^{k}$ such that $(\bar{x}, r, y, z)$ is optimal solution of $\left(F_{r} E D\right)$, since $(\bar{u}, r, y, z)$ is also an optimal solution for $\left(F_{r} E D\right)$, It follows that

$$
f(\bar{x})=f(\bar{u})
$$

by strict pseudoconvexity of $f($.$) we have$

$$
(\bar{x}-\bar{u})^{T} \nabla f(\bar{u})<0
$$

Also from the constraints of $(N E D)$ and $\left(F_{r} E D\right)$ we have $y^{T} g(\bar{x}) \leq y^{T} g(\bar{u})$.

By the semi strictly convexity of $y^{T} g($.$) , this implies$

$$
(\bar{x}-\bar{u}) \nabla y^{T} g(\bar{x}) \leq 0
$$

with strict inequality in the above, if $y_{i}>0$

Also $z^{T} h(\bar{x}) \leq z^{T} h(\bar{u})$ which by quasi-convexity of $z h($.$) at \bar{u}$, implies

$$
(\bar{x}-\bar{u})^{T} \nabla z^{T} h(\bar{u}) \leq 0
$$

Combining (18), (19), and (20), we have

$$
(\bar{x}-\bar{u}) \nabla\left(r f(\bar{u})+y^{T} g(\bar{u})+z^{T} h(\bar{u})\right)<0
$$

which contradicts

$$
(\bar{x}-\bar{u}) \nabla\left(r f(\bar{u})+y^{T} g(\bar{u})+z^{T} h(\bar{u})\right)=0
$$

Hence $\bar{u}=\bar{x}$ i.e. $\bar{u}$ is an optimal solution.

Theorem 5. (Converse Duality): Let $(\bar{x}, r, y, z)$ be an optimal solution of $\left(F_{r} E D\right)$. Assume that

$\left(A_{1}\right): f($.$) is pseudoconvex, y^{T} g($.$) is semi strictly-$ pseudoconvex and $z^{T} h($.$) is quasiconvex.$

$\left(A_{2}\right)$ : Hessian matrix $\nabla^{2}\left(\bar{r} f(\bar{x})+\bar{y}^{T} g(\bar{x})+\bar{z}^{T} h(\bar{x})\right)$ is positive or negative definite, and

$\left(A_{3}\right)$ : the set $\left\{\nabla \bar{y}^{T} g(\bar{x}), \nabla \bar{z}^{T} h(x)\right\}$ is linearly independent, and

Then $\bar{x}$ is an optimal solution of $(N E P)$.

Proof: By Preposition 2, there exist $\tau \in R, \theta \in R^{n}$ $\phi \in R, \psi \in R, \xi \in R$ and $\eta \in R^{m}$ such that

$$
\begin{gathered}
\tau \nabla f(\bar{x})+\theta \nabla^{2}\left(r f(\bar{x})+y^{T} g(\bar{x})+z^{T} h(\bar{x})\right) \\
+\phi \nabla\left(\bar{y}^{T} g(\bar{x})\right)+\psi \nabla z^{T} h(\bar{x})=0 \\
\theta^{T} \nabla f(\bar{x})+\xi=0 \\
\theta \nabla g(\bar{x})+\phi g(\bar{x})+\eta=0 \\
\theta \nabla h(\bar{x})+\psi h(\bar{x})=0 \\
\phi\left(y^{T} g(\bar{x})\right)=0 \\
\psi\left(z^{T} h(\bar{x})\right)=0 \\
\xi r=0 \\
\eta^{T} y=0 \\
(\tau, \phi, \psi, \eta, \xi) \geq 0 \\
(\tau, \theta, \phi, \psi, \eta, \xi) \neq 0 .
\end{gathered}
$$


Multiplying (23) by $y \geq 0$ and using (25) and (28), we obtain

$$
\theta^{T} \nabla y^{T} g(\bar{x})=0
$$

Multiplying (24) by $z$ and $h(\bar{x})=0$, we have

$$
\theta \nabla z^{T} h(\bar{x})=0
$$

Multiplying equality constraint of $\left(F_{r} E D\right)$ by $\theta^{T}$ and using (31) and (32) We have $\theta^{T} \nabla r f(\bar{x})=0$

Multiplying (21) by $\theta$ and using (31) and (32), we have

$$
\tau \theta \nabla f(\bar{x})+\theta \nabla^{2}\left(r f(\bar{x})+y^{T} g(\bar{x})+z^{T} h(\bar{x})\right) \theta=0
$$

Multiplying the above equation by $r$ and using (33), we have

$$
(r \theta)^{T} \nabla^{2}\left(r f(\bar{x})+y^{T} g(\bar{x})+z^{T} h(\bar{x})\right)(r \theta)=0
$$

This because of hypothesis $\left(A_{2}\right)$ implies $r \theta=0$. In view of $\left(A_{3}\right)$ the equality constraint of $\left(F_{r} E D\right)$ implies $r \neq 0$, i.e., $r>0$.consequently $\theta=0$.

Multiplying (21) by $r$ and using $\theta=0$, we have

$$
\tau r \nabla f(\bar{x})+r \phi\left(\nabla y^{T} g(\bar{x})\right)+r \psi\left(\nabla z^{T} h(\bar{x})\right)=0
$$

Using the equality constraint (10) in the above, we have

$$
\begin{aligned}
& -\tau\left(\nabla y^{T} g(\bar{x})+\nabla z^{T} h(\bar{x})\right)+r \phi \nabla y^{T} g(\bar{x}) \\
& +r \psi \nabla z^{T} h(\bar{x})=0
\end{aligned}
$$

This reduces to

$$
(\phi-\tau / r) \nabla y^{T} g(\bar{x})+(\psi-\tau / r) \nabla z^{T} h(\bar{x})=0
$$

By the linear independence hypothesis $\left(A_{3}\right)$. this implies

$$
(\phi-\tau / r)=0 \text { and }(\psi-\tau / r)=0
$$

Now if $\tau=0$, then from above, we have $\phi=0, \psi=0$ and from (22) and (23), We have $\xi=0, \eta=0$, consequently we have $(t, \phi, \psi, \theta, \xi, \eta)=0$ contradicting to (30).

Hence $\tau>0, \phi>0$, and $\psi>0$.

Using $\theta=0$ in (23) and (24), we have

$$
\phi g(\bar{x})+\eta=0, \phi g(\bar{x})+\eta=0
$$

This implies $g(\bar{x}) \leq 0$ and $h(\bar{x})=0$.

Thus $\bar{x}$ is feasible for $\left(F_{r} E D\right)$ and the objective functions of $(N E P)$ and $\left(F_{r} E D\right)$ are equal in their formulations. Under, the state generalized Convexity, Theorem 1 implies that, $\bar{x}$ is an optimal solution of $(N E P)$.

\section{Generalized Fritz John Duality}

Let $M=\{1,2, \cdots, m\}$ and $L=\{1, \cdots, l\}, \quad I_{\alpha} \subseteq M$, $\alpha=0,1, \cdots, t$. with $I_{\alpha} \cap I_{\beta}=\phi, \quad \alpha \neq \beta$ and $\bigcup_{\alpha=0}^{t} I_{\alpha}=M$. and $J_{\alpha} \subseteq L$ with $J_{\alpha} \cap J_{\beta} \neq \phi, \alpha \neq \beta$ and
$L=\bigcup_{\alpha=0}^{t} J_{\alpha}$. Let $K=\{0,1,2, \cdots, t\}$ and $N \subset K$. The following is the generalized Fritz John type dual to $(N E P)$.

$\left(G F_{r} E D\right)$ : Maximize $f(u)$

Subject to

$$
\begin{gathered}
\bar{r} \nabla f(u)+\nabla y^{T} g(u)+\nabla z^{T} h(u)=0 \\
\sum_{i \in I_{\alpha}} y_{i} g_{i}(u) \geq 0, \alpha=0,1,2, \cdots, t \\
\sum_{J \in I_{\alpha}} z_{j} h_{j}(u) \geq 0, \alpha=0,1,2, \cdots, t
\end{gathered}
$$

$$
(\bar{r}, y) \geq 0
$$

$$
(\bar{r}, y, z) \neq 0 .
$$

Theorem 6: If $f($.$) is pseudoconvex, \sum_{i \in I_{\alpha}} y_{i} g($.$) ,$ $\alpha \in N$ is semi-strictly pseudoconvex, $\sum_{i \in I_{\alpha}} y_{i} g($.$) ,$ $\alpha \in K \backslash N$ and $\sum_{j \in I_{\alpha}} z_{j} h_{j}(),. \alpha=0,1,2, \cdots, t$ is quasiconvex,

Then $\inf (N E P) \geq \sup \left(G F_{r} E D\right)$

Proof: Let $x$ be feasible for $(N E P)$ and $(u, r, y, z)$ feasible for $\left(G F_{r} E D\right)$ Suppose $f(x)>f(u)$ This by pseudoconvexity of $f($.$) yields$

$$
\begin{aligned}
& (x-u)^{T} \nabla f(u)<0 \\
& (x-u)^{T} \nabla r f(u) \leq 0
\end{aligned}
$$

with strict inequality in (34) if $r>0$.

From the constraint of $(N E P)$ and $\left(G F_{r} E D\right)$, we have

$$
\sum_{i \in I_{\alpha}} y_{i} g_{i}(x) \leq \sum_{i \in I_{\alpha}} y_{i} g_{i}(u), \alpha \in N
$$

Which because of semistrictly pseudoconvexity of $\sum_{i \in I_{\alpha}} y_{i} g_{i}(x) \alpha=0,1,2, \cdots, t$ implies

$$
(x-u)^{T} \nabla \sum_{i \in I_{\alpha}} y_{i} g_{i}(u) \leq 0, \alpha \in N
$$

with strict inequality in (36) if some $y_{i}>0, i \in I_{\alpha}, \alpha \in N$.

Also

$$
\sum_{i \in I_{\alpha}} y_{i} g_{i}(x)-\sum_{i \in I_{\alpha}} y_{i} g_{i}(u) \leq 0, \alpha \in K \backslash N
$$

And

$$
\sum_{j \in I_{\alpha}} z_{j} h_{j}(x)-\sum_{j \in I_{\alpha}} z_{j} g_{j}(u) \leq 0, \alpha=0,1,2, \cdots, t
$$

Which by quasiconvex of $\sum_{i \in I_{\alpha}} y_{i} g_{i}(),. \alpha \in K \backslash N$ and

$$
\sum_{j \in I_{\alpha}} z_{j} h_{j}(.), \alpha=0,1,2, \cdots, t
$$


respectively imply

$$
(x-u)^{T} \nabla\left(\sum_{i \in I_{\alpha}} y_{i} g_{i}(u)\right) \leq 0, \alpha \in K \backslash N
$$

and

$$
(x-u)^{T} \nabla\left(\sum_{j \in I_{\alpha}} z_{j} h_{j}(u)\right) \leq 0, \alpha \in N
$$

combining (34), (35), (36) and above equation we have

$$
(x-u)^{T}\left(r \nabla f(u)+\nabla y^{T} g(u)+\nabla z^{T} h(u)\right)<0
$$

contradicting the equality constraint of $\left(G F_{r} E D\right)$. Hence $f(x) \geq f(u)$

Implying $\inf (N E P) \geq \sup \left(G F_{r} E D\right)$.

\section{Theorem 7. (Strong Duality):}

If $\bar{x}$ is an optimal solution of $(N E P)$ and there exist $\bar{r} \in R, \bar{y} \in R^{m}$ and $\bar{z} \in R^{k}$, such that $(\bar{r}, \bar{x}, \bar{y}, \bar{z})$ is feasible for $\left(G F_{r} E D\right)$ and the corresponding value of $(N E P)$ and $\left(G F_{r} E D\right)$ are equal. If, the hypotheses of Theorem 1 hold, then $(\bar{x}, \bar{r}, \bar{y}, \bar{z})$ is an optimal solution of $\left(G F_{r} E D\right)$.

Proof: By Proposition 2, there exist $\bar{r} \in R, \bar{y} \in R^{m}$ and $\bar{z} \in R^{k}$ such that

$$
\begin{gathered}
\nabla\left(\bar{r} f(x)+\bar{y}^{T} g(x)+\bar{z}^{T} h(x)\right)=0, \\
\bar{y}^{T} g(\bar{x})=0, \\
(\bar{r}, \bar{y}) \geq 0, \\
(\bar{r}, \bar{y}, \bar{z}) \neq 0
\end{gathered}
$$

Since $\bar{y}_{i} g_{i}(\bar{x})=0, \quad i=1,2, \cdots, m$ and $\bar{z}_{j} h_{j}(\bar{x})=0$, feasibility of $(\bar{r}, \bar{x}, \bar{y}, \bar{z})$ for $\left(G F_{r} E D\right)$ is obvious. Optimality follows, give the pseudoconvexity of $f($.) and semi-strict pseudoconvexity of $\sum_{i \in I_{\alpha}} \bar{y}_{i} g_{i}(),. \alpha \in N$, quasiconvexity of $\sum_{i \in I_{\alpha}} \bar{y}_{i} g_{i}(),. \alpha \in N$, and quasiconvexity of $\sum_{j \in I_{\alpha}} \bar{z}_{j} h_{j}(),. \alpha \in N, \alpha=0,1,2, \cdots, t$ from Theorem 1.

Theorem 8: (Mangasarian [4] Type Strict Converse Duality): Assume that

$\left(A_{1}\right): f($.$) is strictly pseudoconvex,$

$\left(A_{2}\right): \sum_{i \in I_{\alpha}} y_{i} g_{i}(),. \alpha \in N$ is semi-strictly pseudoconvex and

$$
\left(A_{3}\right): \sum_{i \in I_{\alpha}} y_{i} g_{i}(.), \alpha \in K \backslash N \text { and } \sum_{j \in I_{\alpha}} z_{j} h_{j}(.),
$$

$\alpha=0,1,2, \cdots, t$ are quasiconvex.

$\left(A_{4}\right): \bar{x}$ is an optimal solution of $(N E P)$.

If $(\bar{r}, \bar{u}, \bar{y}, \bar{z})$ is an optimal solution of $\left(G F_{r} E D\right)$ then $\bar{x}=\bar{u}$ i.e. $\bar{u}$ is an optimal solution of $(N E P)$.

Proof: Assume that $\bar{x}=\bar{u}$ and exhibit a contradic- tion. Since $\bar{x}$ is an optimal solution of $(N E P)$, by Proposition 2, it implies that there exist $r \in R, y \in R^{m}$ and $z \in R^{k}$ such that $(\bar{x}, r, y, z)$ is an optimal solution of $\left(G F_{r} E D\right)$.

Since $(\bar{u}, r, y, z)$ is an optimal solution for $\left(G F_{r} E D\right)$, it follows that $f(\bar{x})=f(\bar{u})$

This, in view of strict pseudoconvexity of $f($.$) im-$ plies

$$
(\bar{x}-u)^{T} \nabla f(u)<0
$$

From the constraint of $(N E P)$ and $\left(G F_{r} E D\right)$, we have

$$
\sum_{i \in I_{\alpha}} y_{i} g_{i}(\bar{x}) \leq \sum_{i \in I_{\alpha}} z_{i} g_{i}(\bar{u}), \alpha=0,1,2, \cdots, t
$$

and

$$
\sum_{j \in I_{\alpha}} z_{j} h_{j}(\bar{x}) \leq \sum_{j \in I_{\alpha}} z_{j} h_{j}(\bar{u}), \alpha=0,1,2, \cdots, t
$$

The inequality (38), in view of semi-strict pseudo convexity of $\sum_{i \in I_{\alpha}} y_{i} g_{i}(),. \alpha \in N$ implies

$$
(\bar{x}-\bar{u})^{T} \nabla\left(\sum_{i \in I_{\alpha}} y_{i} g_{i}(\bar{u})\right) \leq 0, \alpha \in N
$$

with strict inequality in (40) if $y_{i}>0, i \in I_{\alpha}, \alpha \in N$.

By quasiconvexity of $\sum_{i \in I_{\alpha}} y_{i} g_{i}(),. \alpha \in K \backslash N$, (38) implies

$$
(\bar{x}-\bar{u})^{T} \nabla\left(\sum_{i \in I_{\alpha}} y_{i} g_{i}(\bar{u})\right) \leq 0
$$

The inequality (39), because of quasiconvexity of $\sum_{j \in I_{\alpha}} z_{j} h_{j}(),. \quad \alpha=0,1,2,3, \cdots, t$ yields,

$$
(\bar{x}-\bar{u})^{T} \nabla\left(\sum_{j \in I_{\alpha}} z_{j} h_{j}(\bar{u})\right) \leq 0, \alpha=0,1, \cdots, t
$$

Combining (37), (40), (41) and (42), we have

$$
(\bar{x}-\bar{u})^{T}\left(r \nabla f(\bar{u})+\nabla y^{T} g(\bar{u})+\nabla z^{T} h(\bar{u})\right)<0
$$

which contradicts the feasibility of $(r, \bar{u}, y, z)$ for $\left(G F_{r} E D\right)$. Hence $\bar{x}=\bar{u}$.

Theorem 9 (Converse Duality): Let

$\left(C_{1}\right):(\bar{r}, \bar{x}, \bar{y}, \bar{z})$ be an optimal solution of $\left(G F_{r} E D\right)$.

$\left(C_{2}\right): f($.$) be pseudoconvex, \sum_{i \in I_{\alpha}} \bar{y}_{i} g_{i}(),. \alpha \in N$ semistrictly pseudoconvex, $\sum_{i \in I_{\alpha}} \bar{y}_{i} g_{i}(),. \alpha \in K \backslash N$ quasiconvex.

$\left(C_{3}\right)$ : The Hessian matrix

$$
\nabla^{2}\left(\bar{r} f(\bar{x})+\bar{y}^{T} g(\bar{x})+\bar{z}^{T} h(\bar{x})\right)
$$

is positive or negative definite, and 
$\left(C_{4}\right)$ : The set

$$
\left\{\nabla\left(\sum_{i \in I_{\alpha}} \bar{y}_{i} g_{i}(\bar{x})\right), \nabla\left(\sum_{i \in I_{\alpha}} \bar{z}_{i} h_{i}(\bar{x})\right): \alpha=0,1,2, \cdots, t\right\}
$$

is linearly independent. Then $\bar{x}$ is feasible for $(N E P)$.

Proof: By Proposition 2, there exist $\tau \in R, v \in R^{n}$, $\theta_{\alpha} \in R, \quad \phi_{\alpha} \in R, \quad \alpha=0,1,2, \cdots, t \quad \xi \in R$, and $\eta \in R^{m}$ such that

$$
\begin{gathered}
\tau \nabla f(\bar{x})+v \nabla^{2}\left(r f(\bar{x})+y^{T} g(\bar{x})+z^{T} h(\bar{x})\right) \\
+\sum_{\alpha=0}^{k}\left\{\theta_{\alpha} \nabla\left(\sum_{i \in I_{\alpha}} y_{i} g_{i}(\bar{x})\right)+\sum_{\alpha=0}^{k}\left\{\phi_{\alpha} \nabla\left(\sum_{j \in j_{\alpha}} z_{j} h_{j}(\bar{x})\right)\right\}\right\}=0 \\
v^{T} \nabla f(\bar{x})+\xi=0 \\
v^{T} \nabla g_{i}(\bar{x})+\theta_{\alpha} g_{i}+\eta_{i}=0, i \in I_{\alpha}, \alpha=0,1,2, \cdots, t \\
v \nabla h_{j}(\bar{x})+\phi_{\alpha} h_{j}=0, j \in I_{\alpha}, \alpha=0,1,2, \cdots, t \\
\theta_{\alpha}\left(\sum_{i \in I_{\alpha}} y_{i} g_{i}(\bar{x})\right)=0, \alpha=0,1,2, \cdots, t \\
\phi_{\alpha}\left(\sum_{j \in I_{\alpha}} z_{j} h_{j}(\bar{x})\right)=0, \alpha=0,1,2, \cdots, t \\
\xi^{T} r=0 \\
\eta^{T} y=0 \\
\left(\tau, v, \eta, \xi, \theta_{0}, \theta_{1}, \cdots, \theta_{t}, \phi_{0}, \phi_{1}, \cdots, \phi_{t}\right) \neq 0 \\
\left(\tau, v, \eta, \xi, \theta_{0}, \theta_{1}, \cdots, \theta_{t}\right) \geq 0
\end{gathered}
$$

Multiplying (45) and (46) by $y_{i}$ and $z_{j}$ respectively and using (47) and (48), we have

$$
\begin{aligned}
& v^{T} \nabla\left(\sum_{i \in I_{\alpha}} y_{i} g_{i}(\bar{x})\right)=0 \\
& v^{T} \nabla\left(\sum_{j \in I_{\alpha}} z_{j} h_{j}(\bar{x})\right)=0
\end{aligned}
$$

Multiplying (44) by $r$, we have

$$
r v \nabla f(\bar{x})=0
$$

Multiplying (43) by $v$ and using (53), (54) and (53), we have

$$
\left(r v^{T}\right)\left(r \nabla^{2} f(\bar{x})+\nabla^{2} y^{T} g(\bar{x})+\nabla^{2} z^{T} h(\bar{x})\right)=0
$$

By positive or negative definite and by hypothesis $\left(C_{3}\right)$, we have $r v=0$.

In view of $\left(C_{4}\right)$, equality constraint of $\left(G F_{r} E D\right)$ implies that $r>0$. Hence $v=0$. using $v=0$ we have

$$
\begin{aligned}
& \sum_{\alpha=0}^{r}\left(r \theta_{\alpha}-\tau\right) \nabla\left(\sum_{i \in I_{\alpha}} y_{i} g_{i}(\bar{x})\right) \\
& +\sum_{\alpha=0}^{r}\left(r \phi_{\alpha}-\tau\right) \nabla\left(\sum_{j \in I_{\alpha}} z_{j} h_{j}(\bar{x})\right)=0
\end{aligned}
$$

which in view of the hypothesis $\left(C_{4}\right)$ gives $r \theta_{\alpha}-\tau=0$, $r \phi_{\alpha}-\tau=0, \alpha=0,1,2, \cdots, t$. From (44) and (45), we have $\xi=0$ and $\eta=0$. consequently we have

$$
\left(\tau, v, \eta, \xi, \theta_{0}, \theta_{1}, \cdots, \theta_{t}, \phi_{0}, \phi_{1}, \cdots, \phi_{t}\right)=0,
$$

Contradicting Fritz John Condition (51). Hence $\tau>0$. since $\tau>0, v=0, \eta \geq 0, r>0$, The Equations (45) and (46), implies $g(\bar{x})=0, h(\bar{x})=0$.

Thus $\bar{x}$ is feasible for $(N E P)$ and optimality follows as earlier.

\section{Conclusion}

In this exposition, we have formulated a dual and generalized dual by Fritz John optimality conditions instead of the Karush-Kuhn-Tucker optimality conditions. Consequently no constraint qualification is required and hence such formulations enjoy computational advantage over those formulated by using Karush-Kuhn-Tucker. The problems of these results can be revisited in multiobjective and dynamic setting.

\section{REFERENCES}

[1] R. W. Cottle, "A Theorem of Fritz John in Mathematical Programming," RAND Memorandum RM-3538-PR, 1963.

[2] F. John, "Extremum Problems with Inequalities as Side Condition," In: K. O. Frierichs, O. E. Neugebaur and J. J. Stoker, Eds., Studies and Essays, Courant Anniversary Volume, Wiley (Interscience), New York, 1984, pp. 187204.

[3] T. Weir and B. Mond, "Sufficient Fritz John Optimality Conditions and Duality for Nonlinear Programming Problems," OPSEARCH, Vol. 23, No. 3, 1986, pp. 129-141.

[4] O. L. Mangasarian and S. Fromovitz, "The Fritz John Necessary Optimality Conditions in the Presence of Equality and Inequality Constraints," Journal of Mathematical Analysis and Applications, Vol. 17, No. 1, 1967, pp. 37-47. doi:10.1016/0022-247X(67)90163-1

[5] H. W. Kuhn and A. W. Tucker, "Nonlinear Programming," Proceeding of the Second Berkeley Symposium on Mathematical Statistics and Probability, University of California Press, Berkeley, 1951, pp. 481-492.

[6] O. L. Mangasarian, "Nonlinear Programming," McGrawHill, New York, 1969. 